\title{
Probabilistic discrimination learning of a sequential reinforcement pattern
} STEPHEN E. BUGGIE, I San Jose State
College, San Jose, Calif. 95114

Predictions of the Burke-Estes probabilistic discrimination model were verified in a situation in which reinforcement probabilities varied from .90 to .10 on alternate trials. Fifty college student Ss were able to discriminate between the stimulus traces of reinforcing events of preceding trials in a two-choice light guessing situation. The .90-.10 probabilistic alternation pattern was about three times more difficult to learn than a "pure" 1.00-00 alternation pattern, based on estimates of the discrimination parameter from the data.

Traditionally, discrimination learning has been regarded as a task of distinguishing between two or more stimulating situations which have different component stimuli or different stimulus qualities. However, the statistical learning model (Burke \& Estes, 1957; Estes, Burke, Atkinson, \& Frankmann, 1957) was generalized to "probabilistic discrimination learning," in which two conditions to be discriminated contain the same stimulus components (e.g., the event lights of the typical probability learning apparatus), differing only with respect to the sampling probabilities of the components. An extended theoretical discussion of probabilistic discrimination may be found in Bush \& Mosteller (1951).

Estes \& Burke (1955) proposed an inverse relationship between rate of learning and signal pattern variability; that is, discrimination learning rates (represented by the hypothetical stimulus sampling ratio, $\Theta$ ) would be enhanced when event occurrence probabilities approached extreme values, allowing Ss to predict with good accuracy. For example, rapid discrimination learning would be expected if $\mathrm{II}_{1}$ were near .00 , while $\mathrm{II}_{2}$ were near 1.00 under alternative conditions.

Burke \& Estes (1957) suggested that negatively accelerated response curves approaching respective asymptotes were expected in conditions in which relative proportions of $T_{i}$ stimulating situations are nearly equivalent. When II on odd and even numbered trials is symmetric about .50 , the discrimination parameter $\Theta$ may be estimated by fitting obtained data to the simplified Eq. 1.

$$
P_{n}=1-(1-\theta)^{n-1}
$$

The statistical discrimination model cannot predict beforehand whether discrimination on the basis of stimulus traces of previous reinforcing events will occur in a given situation. ${ }^{2}$ However, if discrimination is obtained, discrimination would be expected to occur more rapidly for a completely deterministic pattern than for a probabilistic pattern which included irregularities. This study investigated the acquisition of a $.90-10$ discrimination between events occurring on alternate trials in a sequence, with comparisons of probabilistic sequences and "pure" alternating pattern sequences within a total pattern of events. Discrimination between odd and even numbered trials, if it occurred, would be evidenced by an increasing trial-by-trial oscillation about the .50 random response level.

\section{METHOD}

The $\mathrm{Ss}$ were 50 naive college students (mean age $=19.1$ ) who were fulfilling a participation requirement for a course in introductory psychology. The Ss were individually run for 60 independent twoalternative trials, using the general procedure and probability learning apparatus described earlier (Buggie, in press). The probabilistic event sequence was individually generated for each $\mathrm{S}$ by using random number tables with Eq. 2.

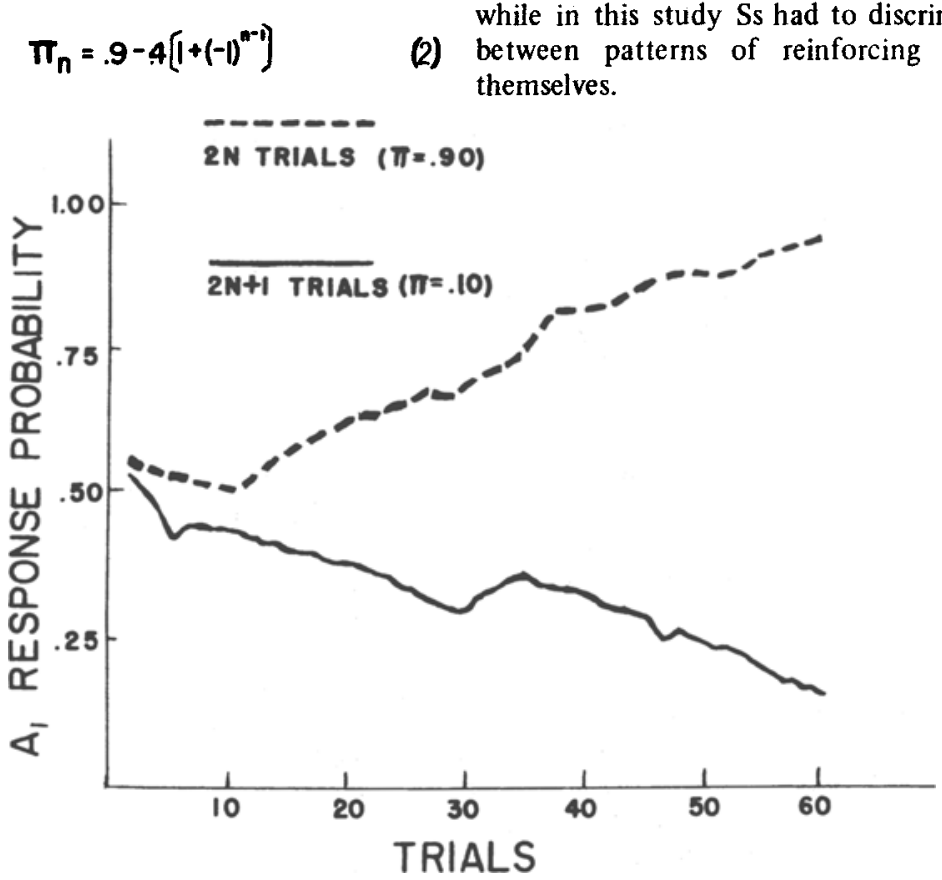

Fig. 1. Acquisition of $\mathbf{. 9 0 - . 1 0}$ probabilistic alternation.
Essentially, the event schedules derived with Eq. 2 yielded a fairly stable E1-E2 trial-by-trial alternation of events, with probability of a sequential disruption on any given trial $=.10$.

RESULTS AND DISCUSSION

The results are summarized in Figs. 1,2, and 3. Data in Fig. 1 were obtained from all Ss over all 60 trials, while data in Figs. 2 and 3 were taken from the subgroup of Ss who had no disruptive event occurrences in their reinforcement patterns for the first 30 trials. Figure 3 was compiled by transforming data from Figs. 1 and 2 for the first 30 trials.

It is apparent that the discrimination between alternate trials was obtained with the probabilistic pattern (Fig. 1) within 60 trials, and even more rapidly for the subgroup of Ss who had a pure alternating event pattern for the first $\mathbf{3 0}$ trials (Fig. 2). Using Eq. 1, conditioning parameter $\Theta$ estimates of .045 (.90-.10 probabilistic alternation) and .142 (1.00-.00 pure alternation) were made. Thus, although discrimination learning occurred among Ss in both reinforcement pattern conditions, the added sequential disruption of the probabilistic schedule made its learning about three times more difficult than the simple, deterministic alternation pattern (Fig. 3). This may imply that even slight probabilistic irregularities in a neardeterministic reinforcement pattern can have quite profound effects on learning rates.

The present study differed from earlier investigations, since previously $\mathrm{Ss}$ were required to discriminate between cues such as signal lights or patterns of signal lights, while in this study Ss had to discriminate between patterns of reinforcing events themselves. 


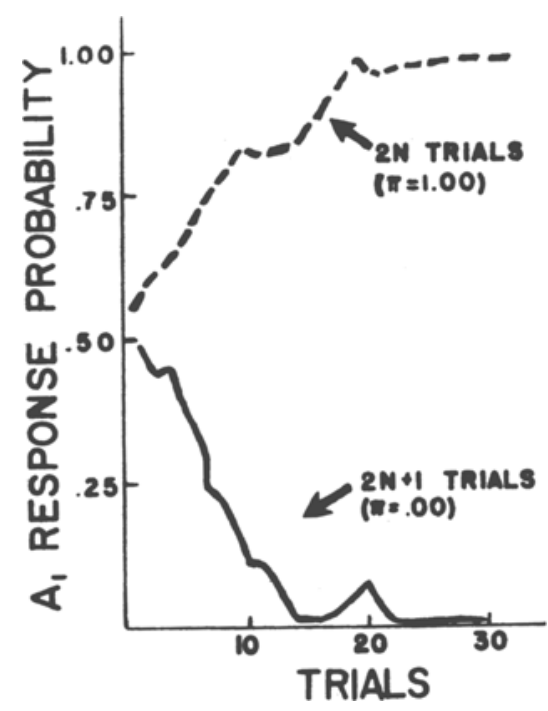

Fig. 2. Acquisition of $1.00-.00$ pure alternation in $\mathbf{3 0}$ trials.

\section{REFERENCES}

BUGGIE, S. E. Probability tracking of a linear periodic reinforcement schedule. Psychonomic Science, in press.

BURKE, C. J., \& ESTES, W. K. A component model for stimulus variables in discrimination learning. Psy chometrika, 1957, 22, 133-145.

BUSH, R. R., \& MOSTELLER, F. A model for stimulus generalization and discrimination. Psychological Review, 1951, 58, 413-423.

ESTES, W. K., \& BURKE, C. J. Application of a

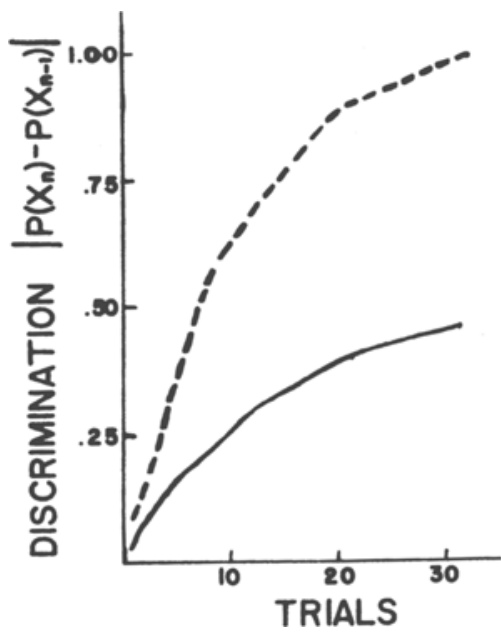

- - - 00-1.00 Pure Alternetion 10- .90 Probubilietice Alternetion

Fig. 3. Comparison of smoothed intertrial discrimination functions from pure and probabilistic sequences.

statistical model to simple discrimination learning in human subjects. Journal of Experimental Psychology, 1955, 50, 81-88.

ESTES, W. K., BURKE, C. J. ATKINSON, R. C., \&

FRANKMANN, J. P. Probabilistic discrimination learning. Journal of Experimental Psychology, 1957, 54, 233-239.

NOTES

1. Now at the University of Oregon, Eugene
Ore. 97403. The author gratefully acknowledges the helpful advice offered by Dr. Robert S. Witte of San Jose State College, and Mr. Charles Bebeau of the University of Colorado. The manuscript was prepared while the author was supported under Contract No. F44620-67-C-0099 between the University of Oregon and the Advanced Research Projects Agency of the Department of Defense. 968

2. Estes, W. K. Personal communication, May

\section{Variability of signal detection measures with noise type}

PATRICK H. McCANN, Naval Personnel Research Activity, San Diego, Calif. 92152

The effects of continuous noise vs intermittent noise on $S s$ performing an audio-visual checking task were examined using TSD (Theory of Signal Detectability) measures of performance. TSD measures were relatively stable for all Ss during both intermittent and continuous noise conditions and closely approximated the values which would be expected in a psychophysical setting. During the last $20 \mathrm{~min}$ of the duty period, there was a corresponding increase in the Ss'response criterion.

An approach to the study of noise effects on vigilance is offered through the theory of signal detectability (Swets, 1964). TSD provides a method for treating false alarm or commission data generated from vigilance tasks. Detection performance is considered as a judgment process in which stimuli are classed by the Os as signals or nonsignals as a function of a criterion that the 0 employs. The criterion is a statistical cut-off between two overlapping normal distributions which represent signal and nonsignal stimulation. An $O$ will utilize a given criterion and will make errors of omission and commission at predictable frequencies. The $O$ 's response criterion (" $\beta$ ") and his discriminative efficiency (" $d$ ") may be computed as measures of vigilance performance. The value $\mathrm{d}^{\prime}$ is the distance in standard scores between the mean of the nonsignal distribution and the mean of the signal distribution. The percentage of detections, $P_{D}$, and percentage of comissions, $P_{C}$, represent areas of signal and nonsignal normal distributions, respectively, for which standard scores may be obtained from a table of normal curve functions. The distance in standard scores of $P_{D}$ to the left of the signal distribution mean plus that of $P_{C}$ to the right of the nonsignal distribution mean equals $d^{\prime}$. The response criterion, $\beta$, is the ratio of the ordinate of the $P_{D}$ point on the signal distribution abscissa to the ordinate of the $\mathrm{P}_{\mathrm{C}}$ point on the nonsignal distribution abscissa. Ordinate values are available from a table of normal curve functions.

Depending upon which noise condition, intermittent or continuous, produces decreased vigilance performance, $d^{\prime}$ and $\beta$ will vary accordingly. Discriminatory efficiency, $d^{\prime}$, should increase and the response criterion, $\beta$, also may increase under the noise condition most conducive to signal detection. The present study tests this hypothesis and examines the relationship between $\beta, \mathrm{d}^{\prime}$, and noise type.

\section{METHOD}

An audio-visual checking task was used upon which the performance of 20 Ss was measured. The task consisted of checking a list of seven-digit numbers against an audio presentation of the numbers. The signal to be detected was a discrepancy between a 